\title{
Qualidade de cortes congelados de tambaqui cultivado
}

\author{
Mônica Maciel Cartonilho(1) e Rogério Souza de Jesus ${ }^{(2)}$
}

\begin{abstract}
(1)Universidade Federal do Amazonas, Avenida General Rodrigo Otávio Jordão Ramos, no 3.000, CEP 69077-000 Manaus, AM. E-mail: monicartonilho@hotmail.com ${ }^{(2)}$ Instituto Nacional de Pesquisas da Amazônia, Coordenação de Tecnologia de Alimentos, Avenida André Araújo, no 2.936, Aleixo, CEP 69060-001 Manaus, AM. E-mail: djesus@inpa.gov.br
\end{abstract}

Resumo - O objetivo deste trabalho foi determinar a composição centesimal, o rendimento, a influência do tempo de congelamento sobre a qualidade e a relação tipo de corte por vida útil, dos cortes de costela, lombinho e posta de tambaqui (Colossoma macropomum) cultivado, durante 180 dias de estocagem. As amostras foram acondicionadas em embalagens de polietileno e congeladas a $-25^{\circ} \mathrm{C}$. Foram realizadas avaliações sensoriais e análises físicoquímicas e microbiológicas dos cortes. $\mathrm{O} \mathrm{pH}$ e os teores de nitrogênio das bases voláteis totais e de substâncias reativas ao ácido tiobarbitúrico, bem como os resultados das análises microbiológicas, foram bons índices do frescor ao longo do tempo de estocagem. O corte de costela foi mais suscetível à oxidação do que os demais; no entanto, os cortes mantiveram-se adequados para o consumo durante todo o experimento, em condições de manipulação correta e com o uso de boas práticas de higiene, entre a colheita e o armazenamento.

Termos para indexação: Colossoma macropomum, estocagem, qualidade, vida útil.

\section{Frozen cut quality of tambaqui reared in fish farms}

\begin{abstract}
The objective of this work was to determine the proximate composition, yield, the influence of frozen storage time on the quality, and the cut type $\mathrm{x}$ shelf life relation of rib, tenderloin and flitch cuts of tambaqui (Colossoma macropomum) reared in a fish farm, during 180 days of cold storage. The cuts were stored in polyethylene bags and frozen at $-25^{\circ} \mathrm{C}$. Sensory, physicochemical and microbiological analyses were carried out. The $\mathrm{pH}$, total volatile basic nitrogen, and thiobarbituric acid-reactive substance values, as well as the results of the microbiological analyses, proved to be good indexes for freshness evaluation along the storage time. The cut rib was more susceptible to oxidation than the other cuts. However, the cuts remained appropriate for consumption during the experiment, under correct handling and good hygienic practices between harvest and storage.
\end{abstract}

Index terms: Colossoma macropomum, storage, quality, shelf life.

\section{Introdução}

A aquicultura é um dos sistemas de produção de alimentos que mais cresce no mundo, e a piscicultura de água doce é a atividade que vem se mostrando mais promissora (Fritsch, 2004). Um fator positivo para o consumidor, com o crescimento da piscicultura, é o equilíbrio entre oferta e demanda no mercado regional, que estabiliza os preços ao longo do ano não só para outras regiões do país, como também para o mercado internacional, ao contrário do que ocorre com a pesca extrativa, em que o preço é regido pela sazonalidade. Outro fator positivo é o desenvolvimento econômico e a geração de emprego para a região produtora (Iglécias, 2007).

O tambaqui (Colossoma macropomum Cuvier, 1818, Characidae, Serrasalminae) é uma das espécies em destaque para o cultivo, por ser originário do Rio Amazonas, estar adaptado às condições da região e pela facilidade para adaptar-se aos ambientes de cultivo (Iziel \& Melo, 2004). Na Região Norte, desponta como a principal espécie de peixe cultivada.

A importância do crescimento da piscicultura na Amazônia e a conquista de novos mercados, particularmente o externo, dependem de sua vinculação ao processo de beneficiamento do pescado, para oferecer produtos que melhor atendam às necessidades e conveniência dos consumidores. Para isso, deve-se ressaltar a necessidade de atendimento rigoroso às normas higiênico-sanitárias, sendo imprescindível a adoção de um programa de análise de perigos e pontos críticos de controle (Paula, 2009). O armazenamento sob condições de congelamento é uma forma de estender a vida útil do pescado; no entanto, a vida de 
prateleira do pescado de água doce congelado tem sido pouco estudada, o que torna importante a realização de trabalhos que para avaliar a estabilidade desses produtos ao longo da armazenagem.

O objetivo deste trabalho foi determinar a composição centesimal dos cortes, a influência do tempo de congelamento na qualidade dos cortes, e a relação tipo de corte $\mathrm{x}$ vida útil do tambaqui.

\section{Material e Métodos}

Foram utilizados cortes de 30 exemplares de tambaqui, capturados no dia 27 de janeiro de $2010 \mathrm{com}$ rede de arrasto, e abatidos por hipotermia, procedentes de piscicultura situada no Município de Itacoatiara, $\mathrm{AM},\left(2^{\circ} 56^{\prime} 40^{\prime \prime} \mathrm{S}\right.$ e $59^{\circ} 15^{\prime} 1$ 'W). O comprimento padrão dos tambaquis, com vísceras e cabeça, variou de 36,50 a $47 \mathrm{~cm}$, com média de $40,23 \pm 2,84 \mathrm{~cm}$. O peso com as vísceras e a cabeça variou de 1,98 a 1,23 kg, com média de $1,54 \pm 0,19 \mathrm{~kg}$.

Após a despesca, os exemplares foram transportados em caixas de isopor com gelo até o laboratório de Tecnologia do Pescado da Coordenação de Pesquisa em Tecnologia de Alimentos, do Instituto Nacional de Pesquisas da Amazônia, em Manaus, AM. A duração do percurso foi de aproximadamente 2 horas e, ao chegarem ao laboratório os animais foram lavados em tanque de inox; em seguida, foram arrumados em camadas de gelo com proporção de 1:1 (uma camada de gelo sobreposta por uma camada de peixe), em caixas de isopor, para proceder à medição, pesagem, evisceração e separação em três tipos de corte (costela, lombinho e posta). Foi utilizada uma balança digital Filizola (Filizola, São Paulo, SP, Brasil) para a medida e pesagem individual dos cortes de tambaqui.

Os padrões de higiene para manipulação de alimentos foram seguidos, e os exemplares de tambaqui foram separados por tipo de corte e colocados em sacos de polietileno de $1 \mathrm{~kg}$. Em seguida, foram submetidos a congelamento rápido, em congelador de placas (Frigoestrela do Brasil, São Paulo, SP, Brasil) a $-36^{\circ} \mathrm{C}$. Após o congelamento, os cortes foram armazenados em freezer Brastemp (Whirlpool Latin America, São Paulo, SP, Brasil) a $-25^{\circ} \mathrm{C}$. As análises sensoriais e físicoquímicas foram realizadas mensalmente durante o período do experimento. A análise microbiológica foi realizada no primeiro dia do experimento e 180 dias depois. O peso médio dos cortes de costela, lombinho e posta foi 207, 292 e 172,5 g respectivamente.

O rendimento dos cortes foi obtido pelo cálculo do volume percentual da parte comestível. As avaliações sensoriais foram realizadas com base na ficha de avaliação de qualidade organoléptica de filés de pescado congelados e descongelados (Tabela 1) (Clucas \& Ward, 1986). As determinações da composição centesimal, valor calórico e $\mathrm{pH}$ dos cortes seguiram as normas analíticas do Instituto Adolfo Lutz (Instituto Adolfo Lutz, 2008) e consistiram das médias de três determinações.

Os peixes foram classificados, de acordo com seu percentual lipídico e proteico, em cinco classes, segundo Stansby (1962), com os seguintes valores: A, baixo teor de gordura, com percentual de gordura inferior a 5 e o de proteína entre 15 e 20; B, semi-gordo, com

Tabela 1. Ficha de avaliação sensorial da qualidade organoléptica de filés de pescado congelado e descongelado, de acordo com classificação proposta por Clucas \& Ward (1986)

\begin{tabular}{|c|c|c|c|c|c|}
\hline Característica & Excelente, 4 & Boa, 3 & Média, 2 & Baixa, 1 & Muito baixa, 0 \\
\hline Cheiro & Sem cheiro & Leve cheiro de peixe & Notável cheiro de peixe & Cheiro de peixe forte & Odor pronunciável \\
\hline Cor da carne & Transparente (branco) & $\begin{array}{l}\text { Transparente tornando-se } \\
\text { translúcido }\end{array}$ & Translúcido & Opaco & Opaco/ cinza/marrom \\
\hline $\begin{array}{l}\text { Transparência } \\
\text { da membrana }\end{array}$ & Transparente & Tornando-se translúcida & Translúcido/ opaca & $\begin{array}{l}\text { Membrana cinza/ } \\
\text { marrom/ opaca }\end{array}$ & $\begin{array}{l}\text { Membrana cinza/ } \\
\text { marrom/ mucosa/pútrida }\end{array}$ \\
\hline Muco & Sem muco & $\begin{array}{l}\text { Levemente mucoso } \\
\text { (muco claro) }\end{array}$ & $\begin{array}{l}\text { Muco notável } \\
\text { (muco claro) }\end{array}$ & $\begin{array}{l}\text { Muito mucoso, com } \\
\text { muco visível (cinza } \\
\text { esverdeado, marrom) }\end{array}$ & $\begin{array}{l}\text { Muito mucoso muco } \\
\text { escuro, opaco }\end{array}$ \\
\hline $\begin{array}{l}\text { Textura e sabor } \\
\text { do filé }\end{array}$ & $\begin{array}{l}\text { Firme elástico (volta } \\
\text { rápido completamente) }\end{array}$ & $\begin{array}{l}\text { Carne firme - mas um pouco } \\
\text { menos elástica (volta, mas } \\
\text { um pouco lentamente) }\end{array}$ & $\begin{array}{l}\text { Carne um pouco mole } \\
\text { (não volta após pressão) }\end{array}$ & $\begin{array}{l}\text { Carne mole - não } \\
\text { retorna após pressão }\end{array}$ & $\begin{array}{l}\text { Carne separando-se, } \\
\text { bem mole }\end{array}$ \\
\hline Danos à carne & Leves manchas, fissuras & $\begin{array}{l}\text { 1-2, defeitos leves (pequenas } \\
\text { fissuras, manchas leves) }\end{array}$ & $\begin{array}{l}\text { 3-5, defeitos leves } \\
\text { (fissuras, manchas leves) }\end{array}$ & $\begin{array}{l}\text { 5, defeitos pequenos } \\
\text { (fissuras, manchas leves) }\end{array}$ & $\begin{array}{l}\text { Filés danificados, } \\
\text { rachados, manchados }\end{array}$ \\
\hline Rachaduras & Sem rachaduras & Rachaduras delicadas & Rachaduras leves & Rachaduras moderadas & Rachaduras severas \\
\hline
\end{tabular}

${ }^{(1)}$ Texto traduzido para este trabalho. 
percentual de gordura e de proteína entre 5 e 15; C, alto teor de gordura, com percentual de gordura maior que 15 e o proteico menor que 15 ; $\mathrm{D}$, baixo teor de gordura e alto teor proteico, com percentual de gordura menor que 5 e o de proteína maior que 20; e E, baixo teor de gordura e baixo teor proteico, com percentual de gordura menor que 5 e o de proteína menor que 15. Considerando-se o percentual lipídico e o valor energético, o pescado foi classificado segundo Almás (1981), em três classes: magro, percentual lipídico entre 0,2 e 0,8 , e valor energético entre 80 e $90 \mathrm{kcal}$ por 100 g; semigordo, percentual lipídico entre 2 e 5,7, e valor energético entre 90 e $160 \mathrm{kcal}$ por $100 \mathrm{~g}$; e gordo, percentual lipídico entre 8 e 14, e valor energético entre 160 e $220 \mathrm{kcal}$ por $100 \mathrm{~g}$.

As análises para a determinação de nitrogênio das bases voláteis totais (N-BVT) foram realizadas em triplicata para cada corte, a cada 30 dias, conforme método de Wootlon \& Chuah (1981), adaptado por Jesus (1998). A estabilidade oxidativa das substâncias reativas ao ácido tiobarbitúrico (TBARS) foi determinada de acordo com Vyncke (1970), adaptada quanto ao tempo de reação entre o extrato e o ácido tiobarbitúrico (TBA) sob temperatura de ebulição que foi reduzido de 40 para $30 \mathrm{~min}$.

A metodologia utilizada para as análises microbiológicas (contagem de coliformes totais e mesófilos) seguiu as normas estabelecidas por Silva et al. (2007). Os resultados foram comparados aos padrões microbiológicos da Agência Nacional de Vigilância Sanitária (Agência Nacional de Vigilância Sanitária, 2001).

$\mathrm{Na}$ análise estatística, foi estabelecida uma matriz de correlação entre as variáveis por meio do programa Action 1.0, a 5\% de probabilidade. A determinação das TBARS somente foi iniciada aos 90 dias de armazenamento, em razão da necessidade de obtenção de um maior número de dados, que certificassem a qualidade dos cortes pelo período de 180 dias de armazenamento em condições de congelamento.

\section{Resultados e Discussão}

O rendimento obtido dos cortes de tambaqui foi de $19,64 \%$ para costela, de $27,70 \%$ para lombinho, e de $16,37 \%$ para posta. O rendimento total foi de $63,71 \%$.

Os valores sensoriais (Figura 1) variaram de 28 pontos, para todos os cortes, durante os primeiros 30 dias de armazenamento congelado, a 23 pontos aos 180 dias de experimento. Esses valores indicam que os cortes de tambaqui permaneceram sempre em qualidade excelente, de acordo com Clucas \& Ward (1986).

Ao analisar a composição centesimal do tambaqui (Tabela 2), o corte de costela, segundo Stansby (1962), pode ser classificado como de peixe de classe B (semigordo), e os cortes de lombinho e posta como peixe de classe A (baixo teor de gordura). Considerando-se a classificação de Almás (1981), os cortes de costela, lombinho e posta classificamse como de pescado semigordo. As variações mais expressivas foram nos teores de gordura e umidade, e o corte de costela apresentou o maior teor. Segundo Arbelaéz-Rojas et al. (2002), a quantidade de gordura influencia positivamente a aceitação do peixe pelo mercado consumidor, que se justifica porque os lipídeos associados aos demais constituintes melhoram a palatabilidade e conferem sabor mais agradável à carne.

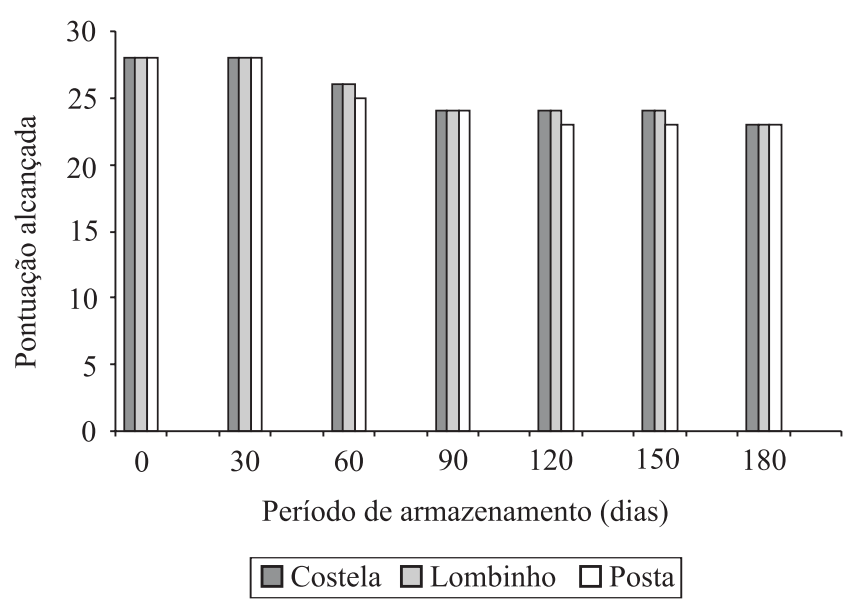

Figura 1. Avaliação sensorial e pontuação final obtidas ao longo de 180 dias sob condições de congelamento, em cortes de costela, lombinho e posta de tambaqui (Colossoma macropomum).

Tabela 2. Composição centesimal dos cortes in natura de tambaqui (Colossoma macropomum) cultivado.

\begin{tabular}{lccc}
\hline Composição (\%) & \multicolumn{1}{c}{ Costela } & Lombinho & \multicolumn{1}{c}{ Posta } \\
\hline Umidade & $71,27 \pm 1,33$ & $77,49 \pm 0,46$ & $77,65 \pm 0,54$ \\
Lipídeos & $7,69 \pm 0,04$ & $1,59 \pm 0,25$ & $2,18 \pm 0,04$ \\
Proteína & $19,80 \pm 0,15$ & $19,63 \pm 0,21$ & $18,85 \pm 0,09$ \\
Cinza & $1,12 \pm 0,07$ & $1,14 \pm 0,02$ & $1,19 \pm 0,01$ \\
Glicídeos $^{(1)}$ & 0,12 & 0,15 & 0,13 \\
Energia (kcal por $100 \mathrm{~g})$ & 148,89 & 93,43 & 96,54 \\
\hline
\end{tabular}

${ }^{(1)}$ Cálculo obtido por diferença. 
Porém, os lipídeos podem influenciar negativamente a qualidade final, em consequência de mudanças degradativas pós-morte, que ocorrem de forma mais rápida em peixes com maiores concentrações lipídicas. Esse fato pode levar a uma redução no tempo de vida de prateleira, principalmente em decorrência das reações oxidativas durante o armazenamento.

Os valores de $\mathrm{pH}$ nos cortes de tambaqui foram crescentes no decorrer dos 180 dias de congelamento. Os valores de $\mathrm{pH}$ dos cortes de costela, lombinho e posta foram, ao início do experimento, de 5,60, 5,58 e 5,61, respectivamente, e de 6,70, 6,68 e 6,66 respectivamente, aos 180 dias de estocagem sob congelamento a $-25^{\circ} \mathrm{C}$.

O Regulamento de Inspeção Industrial e Sanitária de Produtos de Origem Animal - RIISPOA (Brasil, 2001) estabelece os seguintes limites máximos de $\mathrm{pH}$ para que um peixe possa ser considerado como pescado fresco: $\mathrm{pH}$ inferior a 6,8 na carne externa e a 6,5 na carne interna. Oehlenschläger \& Söresen (1997) afirmam que o $\mathrm{pH}$ do pescado fresco varia de 6,6 a 6,8 , e à medida que o peixe se deteriora, os valores de $\mathrm{pH}$ aumentam e podem atingir 7,2; esses autores consideram que o $\mathrm{pH}$ de um peixe fresco é menor que 7.

Os valores de o N-BVT dos cortes de tambaqui cultivado, durante 180 dias de estocagem a $-25^{\circ} \mathrm{C}$, foram crescentes até os 90 dias (Figura 2), seguidos de oscilações decrescentes no período compreendido entre 90 e 150 dias. Após esse período, observouse aumento dos valores de N-BVT até o final do experimento, que não alcançaram o limite de $30 \mathrm{mg}$ de N-BVT por100 g.

Segundo Ogawa \& Ogawa (1999), os valores de N-BVT, em peixes eviscerados e congelados, foram mais adequados, provavelmente em consequência da diminuição das enzimas do tecido visceral que aceleram a autólise. De acordo com o limite máximo, estabelecido pelo Ministério da Agricultura, Pecuária e Abastecimento (Brasil, 2001), os cortes do presente trabalho foram manipulados com segurança adequada para o consumo, durante todo o período de estocagem, pois os valores máximos de N-BVT obtidos, para cada corte, situaram-se bem abaixo do limite estabelecido.

Os valores de TBARS foram utilizados como indicadores do grau de oxidação lipídica, que quantificam o malonaldeído, um dos principais produtos formados durante o processo oxidativo. Os valores de TBARS foram crescentes ao longo do tempo de armazenamento, e os maiores valores foram obtidos no corte de costela (Figura 2). Esse corte apresentou maior teor lipídico que os demais, o que o tornou mais susceptível a oxidação, fato que constitui um entrave para armazenagem por longo tempo, quanto à qualidade nutricional, organoléptica e microbiológica adequada.

Os resultados das análises de TBARS confirmam a afirmação de Sant'ana \& Mancini-Filho (2000) de que somente o abaixamento da temperatura não é suficiente para impedir o processo de desenvolvimento da oxidação lipídica, que ocorre mesmo durante a estocagem de alimentos congelados, pois o armazenamento congelado não paralisa as reações oxidativas. Para obtenção do aumento do tempo de vida útil de cortes congelados, o armazenamento com embalagem a vácuo tem sido estudado.

Weber (2007), em sua pesquisa, analisou entre outros fatores, a influência da embalagem a vácuo sobre a estabilidade lipídica de filés crus, congelados
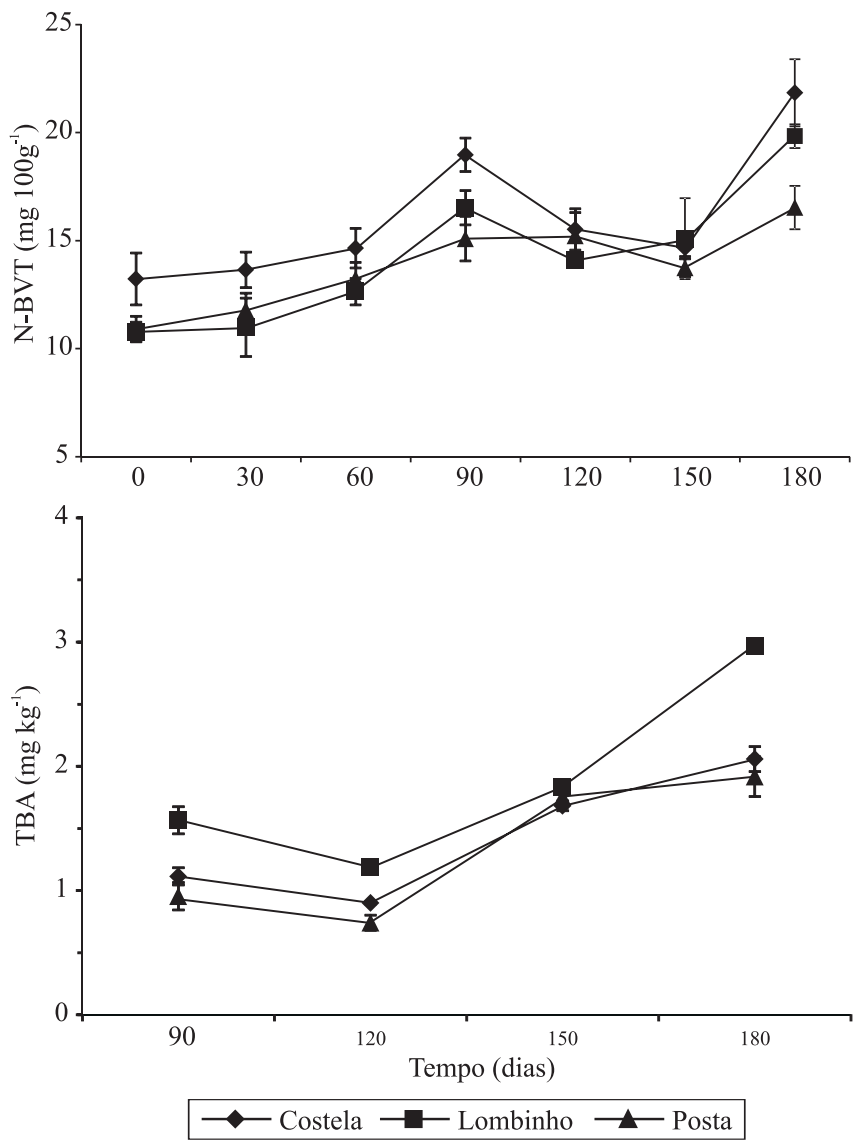

Figura 2. Valores de nitrogênio das bases voláteis totais (N-BVT) e de substâncias reativas ao ácido tiobarbitúrico (TBA) em cortes de tambaqui (Colossoma macropomum) cultivado, armazenados sob condições de congelamento durante 180 dias. 
por 18 meses, e observou que os filés avaliados foram considerados aptos para o consumo, pois o aumento dos valores de TBARS ocorreu ao décimo segundo mês de estocagem congelada. Com base no índice descrito por Al Kahtani et al. (1996), Weber (2007) afirma que os filés avaliados poderiam ser considerados aptos para o consumo por mais de 18 meses após o congelamento.

No presente trabalho, os valores de TBARS para os cortes de tambaqui estavam abaixo de $3 \mathrm{mg} \mathrm{kg}^{-1}$ de malonaldeido, o que indica que o peixe estava em bom estado de conservação, sem odor e sabor característicos de ranço, até os 180 dias sob estocagem congelada. Certamente, valores menores de TBARS poderiam ter sido obtidos, se a embalagem fosse feita a vácuo.

Durante os 180 dias de estocagem congelada, os diferentes cortes analisados (costela, lombinho e posta) apresentaram valores de coliformes totais e mesófilos a $35^{\circ} \mathrm{C}$ relativamente baixos (Tabela 3 ), com ausência de coliformes termo-tolerantes, psicrófilos, bolores e leveduras. Portanto, não houve comprometimento da qualidade dos cortes, o que garantiu que apresentavam boa qualidade higiênico-sanitária, de acordo com a legislação (Agência Nacional de Vigilância Sanitária, 2001). Ao final do experimento, foram obtidos valores decrescentes para as contagens microbiológicas, em comparação aos valores obtidos antes do congelamento, para todos os cortes, comportamento este típico em condições de congelamento. Isto era esperado, em razão da baixa temperatura que leva à paralisação do desenvolvimento microbiano. Tais resultados foram semelhantes aos obtidos por Jesus (1998), em sua pesquisa com "minced fish" de diferentes espécies amazônicas.

De acordo com o estabelecido pela atual legislação (Agência Nacional de Vigilância Sanitária, 2001) quanto a coliformes termo-tolerantes em pescado in natura, resfriado ou congelado, não consumido cru, a ausência desses microrganismos no presente trabalho, antes e após a armazenagem sob congelamento por 180 dias, indica que os cortes analisados apresentavam boas condições higiênicas.

Os resultados obtidos indicam a eficiência do congelamento em reduzir o crescimento dos microrganismos, em consequência do tempo de armazenagem, o que constitui uma forma de estender a vida útil de pescados.

De acordo com os resultados das correlações entre as análises realizadas nos cortes de tambaqui estocado a $-25^{\circ} \mathrm{C}$, durante 180 dias, a $5 \%$ de probabilidade para todas as variáveis (Tabela 4), os valores de $\mathrm{pH}$ dos

Tabela 3. Contagem microbiológica de cortes de tambaqui (Colossoma macropomum), frescos e após 180 dias de armazenagem sob congelamento.

\begin{tabular}{|c|c|c|c|c|}
\hline \multirow[t]{2}{*}{ Corte } & \multicolumn{2}{|c|}{ Fresco } & \multicolumn{2}{|c|}{ Após 180 dias } \\
\hline & Coliformes totais $\left(\mathrm{NMP} \mathrm{g}^{-1}\right)$ & Aeróbios mesófilos $\left(\mathrm{UFC} \mathrm{g}^{-1}\right)$ & Coliformes totais $\left(\mathrm{NMP} \mathrm{g}^{-1}\right)$ & Aeróbios mesófilos $\left(\mathrm{UFC} \mathrm{g}^{-1}\right)$ \\
\hline Costela & $2,30 \pm 0,5 \times 10$ & $4,90 \pm 1,3 \times 10$ & $1,10 \pm 0,1 \times 10$ & $2,40 \pm 0,5 \times 10$ \\
\hline Lombinho & $2,30 \pm 0,3 \times 10$ & $3,13 \pm 1,03 \times 10^{2}$ & $1,30 \pm 0,3 \times 10$ & $2,30 \pm 0,3 \times 10^{2}$ \\
\hline Posta & $2,40 \pm 0,55 \times 10^{2}$ & $1,57 \pm 0,37 \times 10^{2}$ & $1,90 \pm 0,4 \times 10^{2}$ & $1,57 \pm 0,37 \times 10^{2}$ \\
\hline
\end{tabular}

Tabela 4. Matriz de correlação de Pearson, entre as variáveis analisadas, para cortes de costela, lombinho e posta de tambaqui (Colossoma macropomum) cultivado.

\begin{tabular}{|c|c|c|c|c|c|c|c|c|c|c|}
\hline Variáveis & Tempo & $\begin{array}{c}\mathrm{pH} \\
\text { costela }\end{array}$ & $\begin{array}{c}\mathrm{pH} \\
\text { lombinho }\end{array}$ & $\begin{array}{c}\mathrm{pH} \\
\text { posta }\end{array}$ & $\begin{array}{l}\text { N-BVT } \\
\text { costela }\end{array}$ & $\begin{array}{c}\text { N-BVT } \\
\text { lombinho }\end{array}$ & $\begin{array}{c}\text { N-BVT } \\
\text { posta }\end{array}$ & $\begin{array}{c}\text { Sensorial } \\
\text { costela }\end{array}$ & $\begin{array}{l}\text { Sensorial } \\
\text { lombinho }\end{array}$ & $\begin{array}{c}\text { Sensorial } \\
\text { posta }\end{array}$ \\
\hline Tempo & 1 & & & & & & & & & \\
\hline $\mathrm{pH}$ costela & $0,951 *$ & 1 & & & & & & & & \\
\hline pH lombinho & $0,963 *$ & $0,999^{\text {ns }}$ & 1 & & & & & & & \\
\hline $\mathrm{pH}$ posta & $0,949 *$ & $0,989^{\text {ns }}$ & $0,987^{\mathrm{ns}}$ & 1 & & & & & & \\
\hline N-BVT costela & $0,701^{\mathrm{ns}}$ & $0,673^{\mathrm{ns}}$ & $0,684^{\mathrm{ns}}$ & $0,665^{\mathrm{ns}}$ & 1 & & & & & \\
\hline N-BVT lombinho & $0,881 *$ & $0,847^{*}$ & $0,858 *$ & $0,834 *$ & $0,945^{*}$ & 1 & & & & \\
\hline N-BVT posta & $0,879 *$ & $0,913 *$ & $0,919 *$ & $0,914 *$ & $0,859 *$ & $0,914^{*}$ & 1 & & & \\
\hline Sensorial costela & $-0,918 *$ & $-0,965^{*}$ & $-0,968 *$ & $-0,967 *$ & $-0,612^{\mathrm{ns}}$ & $-0,797 *$ & $-0,895^{*}$ & 1 & & \\
\hline Sensorial lombinho & $-0,936^{*}$ & $-0,973 *$ & $-0,978 *$ & $-0,960 *$ & $-0,761 *$ & $-0,904 *$ & $-0,950 *$ & $0,974 *$ & 1 & \\
\hline$\underline{\text { Sensorial posta }}$ & $-0,918^{*}$ & $-0,965^{*}$ & $-0,968 *$ & $-0,967^{*}$ & $-0,612^{\mathrm{ns}}$ & $-0,797^{*}$ & $-0,895^{*}$ & $1 *$ & $0,974 *$ & 1 \\
\hline
\end{tabular}

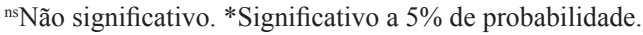


cortes de costela, lombinho e posta apresentaram bons indicadores da qualidade em razão do tempo nessas condições de armazenamento.

Os valores de N-BVT dos cortes de lombinho e posta tiveram correlação significativa em consequência do tempo. No entanto, a correlação entre os valores de N-BVT do corte de costela e o tempo não foi significativa, o que indica que este corte foi mais instável. Isso pode ser explicado pelo seu alto teor lipídico, que o torna mais suscetível à oxidação formada durante o período de estocagem. Assim, também, observou-se correlação significativa entre N-BVT e $\mathrm{pH}$, para os cortes de lombinho e de posta, e correlação não significativa para os cortes de costela. Os escores sensoriais para todos os cortes tiveram correlação significativa em razão do tempo. Pode-se, ainda, observar correlação significativa entre o escore sensorial e N-BVT, escore sensorial e o $\mathrm{pH}$, para os cortes de lombinho e posta. A correlação entre escore sensorial e N-BVT da costela não foi significativa.

\section{Conclusões}

1. A oxidação é prejudica o armazenamento sob congelamento de cortes de tambaqui por períodos acima de 180 dias, em embalagem de polietileno.

2. Os cortes de costela de tambaqui armazenados sob congelamento são menos estáveis à oxidação, em razão de sua elevada concentração de lipídios.

3. Os valores do $\mathrm{pH}$, das bases voláteis totais e das substâncias reativas ao àcido tiobarbitúrico de cortes de tambaqui armazenados sob congelamento são bons índices de avaliação do frescor.

4. Os cortes de tambaquis armazenados sob congelamento apresentam índice decrescente de crescimento de microrganismos em razão do tempo.

5. O congelamento por 180 dias é eficiente para retardar o processo de deterioração e estender a vida útil dos cortes de tambaqui.

\section{Referências}

AGÊNCIA NACIONAL DE VIGILÂNCIA SANITÁRIA. Resolução RDC no 12 , de 2 de janeiro de 2001. Regulamento técnico sobre os padrões microbiológicos para alimentos. Diário Oficial [da] República Federativa do Brasil, 4 jan. 2001.

AL-KAHTANI, H.A.; ABU-TARBOUSH, H.M.; BAJABER, A.S.; ATIA, M.; ABOU-ARAB, A.A.; EL-MOJADDIDI, M.A. Chemical changes after irradiation and post irradiation storage in tilapia and Spanish mackerel. Journal of Food Science, v.61, p.729-733, 1996.

ALMÁS, K.A. Chemical and microbiology of fish and fish processing. Norway: University of Throndhein, 1981. 123p.

ARBELAÉZ-ROJAS, G.A.; FRACALOSSI, D.M.; FIM, J.D.I. Composição corporal de tambaqui, Colossoma macropomum, e matrinxã, Bryconcephalus, em sistemas de cultivo intensivo, em igarapé, e semi-intensivo, em viveiros. Revista Brasileira de Zootecnia, v.3, p.1059-1069, 2002.

BRASIL. Ministério da Agricultura, Pecuária e Abastecimento. Regulamento da inspeção industrial e sanitária de produtos de origem animal-RIISPOA: pescados e derivados. 2001. Disponível em:__ <http://www.agricultura.gov.br/arq_editor/file/Aniamal/ MercadoInterno/Requisitos/RegulamentoInspecaoIndustrial.pdf $>$. Acesso em: 10 jul. 2010.

CLUCAS, J.J.; WARD, A.R. Post-harvest fisheries development: a guide to handling, preservation, processing and quality. Chatham: Natural Resources Institute, 1986. 443p.

FRITSCH, J. A hora e a vez do peixe. Higiene Alimentar, v.18, n.116/117, 2004.

IGLÉCIAS, W. O empresariado do agronegócio no Brasil: ação coletiva e formas de atuação política - as batalhas do açúcar e do algodão na OMC. Revista de Sociologia e Política, v.28, p.75-97, 2007.

INSTITUTO ADOLFO LUTZ. Normas analíticas do Instituto Adolfo Lutz: métodos químicos e físicos para análises de alimentos. 4.ed. São Paulo: Instituto Adolfo Lutz, 2008. 1002p.

IZEL, A.C.U.; MELO, L.A.S. Criação de tambaqui (Colossoma macropomum) em tanques escavados no Estado do Amazonas. Manaus: Embrapa Amazônia Ocidental, 2004. 20p. (Embrapa Amazônia Ocidental. Documentos, 32).

JESUS, R.S. de. Estabilidade de "minced fish" de peixes amazônicos durante o congelamento. 1998. 105p. Dissertação (Doutorado) - Universidade de São Paulo, São Paulo.

OEHLENSCHLÄGER, J.; SÖRENSEN, N.K. Criteria of fish freshness and quality aspects. In: THE FINAL MEETING OF THE CONCERTED ACTION "EVALUATION OF FISH FRESHNESS", 1997, Nantes. Proceedings. Paris: International Institute of Refrigeration, 1997. p.30-35.

OGAWA, M.; OGAWA, N.B. Alterações do pescado post-mortem. In: OGAWA, M.; MAIA, E.L. (Ed.). Manual de pesca. São Paulo: Varela, 1999. p.175-189.

PAUlA, F.G. de. Desempenho do tambaqui (Colossoma macropomum), de pirapitinga (Piaractus brachypomum), e do híbrido tambatinga (C. macropomum x $P$. brachypomum) mantidos em viveiros fertilizados, na fase de engorda. 2009. 57p. Dissertação (Mestrado) - Universidade Federal de Goiás, Goiânia.

SANT'ANA L.S.; MANCINI-FILHO, J. Influence of the addition of antioxidants in vivo on the fatty acid composition of fish fillets. Food Chemistry, v.68, p.175-178, 2000.

SILVA, N. da; JUNQUEIRA, V.C.A.; SILVEIRA, N.F. de A.; TANIWAKI, M.H.; SANTOS, R.F.S. dos; GOMES, R.A.R. Manual de métodos de análise microbiológica de alimentos. 3.ed. São Paulo: Varela, 2007. 536p. 
STANSBY, M.E. Proximate composition of fish. In: HEEN, E.; KREUEER, R. (Ed.). Fish in nutrition. London: Fishing News, 1962. p.55-60.

VYNCKE, W. Direct determination of the thiobarbituric acid value in trichloroacetic acid extracts of fish as a measure of oxidative rancidity. Fette Seifen Anstrichmittel, n.12, p.1084-1087, 1970.
WEBER, J. Estabilidade lipídica de filés de jundiá (Rhamdia quelen). 2007. 81p. Dissertação (Mestrado) - Universidade Federal de Santa Maria, Santa Maria.

WOOTLON, M.; CHUAH, S.H. The use of sea mullet (Mugil cephalus) in the production of cold marinades. Food Technology in Australia, v.33, p.392-397, 1981.

Recebido em 4 de outubro de 2010 e aprovado em 4 de fevereiro de 2011 